\title{
DEM L241, a Supernova Remnant containing a High-Mass X-ray Binary
}

\author{
F. D. Seward \\ Smithsonian Astrophysical Observatory, 60 Garden St., Cambridge MA 02138, USA \\ P. A. Charles \\ School of Physics and Astronomy, University of Southampton, Highfield, Southampton, \\ SO17 1BJ,UK and Department of Astronomy, University of Cape Town, Private Bag X3, \\ Rondebosch 7701, Republic of South Africa \\ D. L. Foster \\ South African Astronomical Observatory, P.O. Box 9, Observatory 7935, Cape Town, \\ South Africa and Vanderbilt University, Department of Physics and Astronomy, Nashville,
} TN 37235

J. R. Dickel

P. S. Romero

Department of Physics and Astronomy, University of New Mexico, 1919 Lomas Blvd. NE, Albuquerque, NM 87131, USA

Z. I. Edwards

M. Perry

R. M. Williams

Department of Earth and Space Sciences, Columbus State University, Coca Cola Space Science Center, 701 Front Avenue, Columbus, GA 31901, USA

Received ; accepted 


\begin{abstract}
A Chandra observation of the Large Magellanic Cloud supernova remnant DEM L241 reveals an interior unresolved source which is probably an accretionpowered binary. The optical counterpart is an O5III(f) star making this a HighMass X-ray Binary (HMXB) with orbital period likely to be of order tens of days. Emission from the remnant interior is thermal and spectral information is used to derive density and mass of the hot material. Elongation of the remnant is unusual and possible causes of this are discussed. The precursor star probably had mass $>25 M_{\odot}$
\end{abstract}

keywords: supernova remnants-Magellanic Clouds-X-rays:binaries-X-rays:individual(DEM L241)

\title{
1. Introduction
}

In the Magellanic Clouds there are now 34 supernova remnants known to emit X-rays. Ten of these have interior pulsar-wind nebulae (PWNe) or compact objects which also radiate in the X-ray band. Although the sample is small, there is great diversity. Several manifestations of neutron stars are represented and the present observation may be an example of yet another.

X-rays from the supernova remnant SNR 0535-67.5 in the H II region DEM L241 (Davis et al 1976) were first detected in 1979 by Long et al (1981). The supernova remnant was first identified by Mathewson et al (1985) and mapped using the optical [S II] emission as seen in Figure 1 which shows the remnant and the surrounding H II emission. Since the initial Einstein observation there have been X-ray detections by ROSAT (Williams et al. 1999), XMM (Bamba et al. 2006), and now Chandra. The XMM data provided the 
first detailed X-ray image and showed elongated diffuse emission filling the area outlined by [SII] filaments with a bright hard point source centered in the SE section. Because the spectrum of this source was a power law, Bamba et al (2006) identified it as an unresolved PWN. The Chandra observation was planned to resolve this object and to distinguish the expected point-like pulsar from surrounding diffuse emission. The Chandra result indeed shows a clear point-like hard source but there is no sign of a PWN close to or surrounding the point source.

\section{The Chandra Observation}

Chandra observed DEM L241 on 2011 February 7 and 8 for 46 ks with the ACIS detector. The observation was in two continuous parts of length 22 and 24 ks (OBSIDs 12675 and 13226) separated by an interval of $40 \mathrm{ks}$. Figures 2 and 33 show the result. The supernova remnant has dimension $2.2^{\prime} \times 5.3^{\prime}$ with diffuse emission filling the interior and with no limb brightening from an outer shell. The remnant appears to be almost divided into two parts. The southern section, called the "Head" by Bamba et al (2006) has dimension approximately $1.4^{\prime} \times 1.8^{\prime}$ with the bright compact source somewhat off-center. The elongated northern part, called the "Tail" has dimension $2.2^{\prime} \times 3.7^{\prime}$. The entire X-ray

remnant fits within and follows the [S II] filaments seen in Figure 1. The compact source, being close to the brightest diffuse emission, appears to be associated with the remnant but its characteristics are unusual, as will be discussed in the next section. 


\section{The Compact Source}

\subsection{X-rays}

The compact X-ray source appears point-like and has a luminosity $\sim 2 \times 10^{35} \mathrm{erg} \mathrm{s}^{-1}$ at the LMC distance of $50 \mathrm{kpc}$. No extended PWN is visible in the immediate vicinity and the diffuse emission close to the point source is thermal. As shown in Figure 4, the Chandra telescope PSF (Point Spread Function) accounts very well for the appearance of the source. The source X-ray spectrum is a power law and it is appreciably harder than that from the diffuse parts of the remnant.

It is very unlikely that the object is a background AGN. There are an average of 0.2 AGN deg ${ }^{-2}$ at this flux level (Kim et al 2007) so the chance of finding one even within the $2^{\prime} \times 5^{\prime}$ remnant is only $\approx 6 \times 10^{-4}$. Furthermore the $\mathrm{X}$-ray spectral index is -1.3 which is harder than the mean index of -1.9 (with dispersion of 0.5 ) for radio quiet QSOs.

The X-ray spectrum is also too hard to be that of a foreground normal star or even that of the optical counterpart O5III(f) star. This star itself is expected to be an X-ray source but with a soft spectrum and luminosity only $1 \%$ of the flux we observe here (Chlebowski 1989). Colliding winds in a binary system could be more energetic but the luminosity of observed systems (e.g. W140 - Pollock et al 2005) is still an order of magnitude less than that of this object. We conclude that the source is neither a foreground nor a background object and is probably associated with the remnant.

The Chandra X-ray spectrum is shown in Figure 5. Data are from a circular region with radius $4^{\prime \prime}$ centered on the source and background was taken from a surrounding $5^{\prime \prime}$ wide annulus. The count rate was $0.057 \mathrm{~s}^{-1}$ which, in the ACIS instrument which integrates for $3.2 \mathrm{~s}$, leads to a $3 \%$ chance of two events being recorded as one. This pileup is not severe but enough to distort the spectrum. We therefore incorporated a pileup correction, 


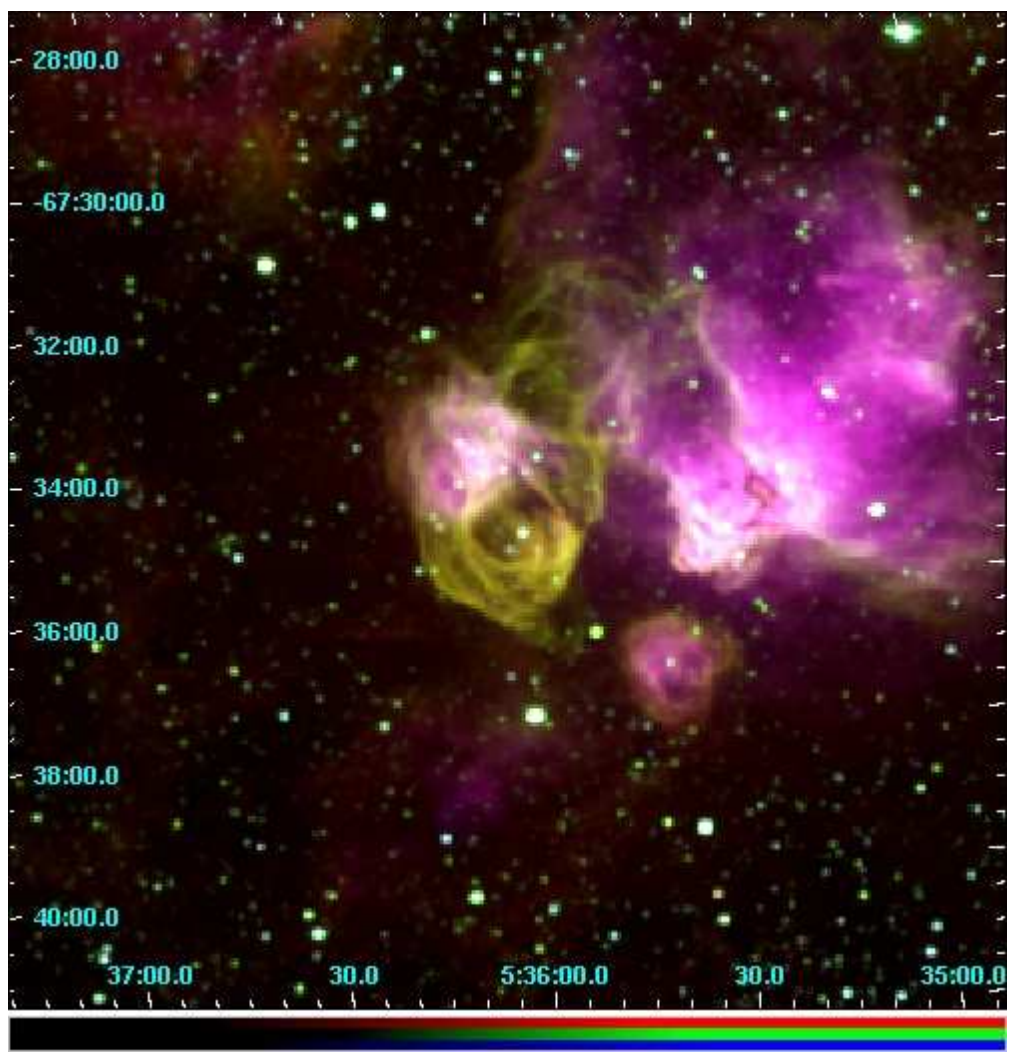

Fig. 1.- The H II region DEM L241 showing H emission in red and [S II] emission in yellow. The [S II] emission defines the supernova remnant and correlates well with the X-rays. Figure from Smith et al 1999 . 


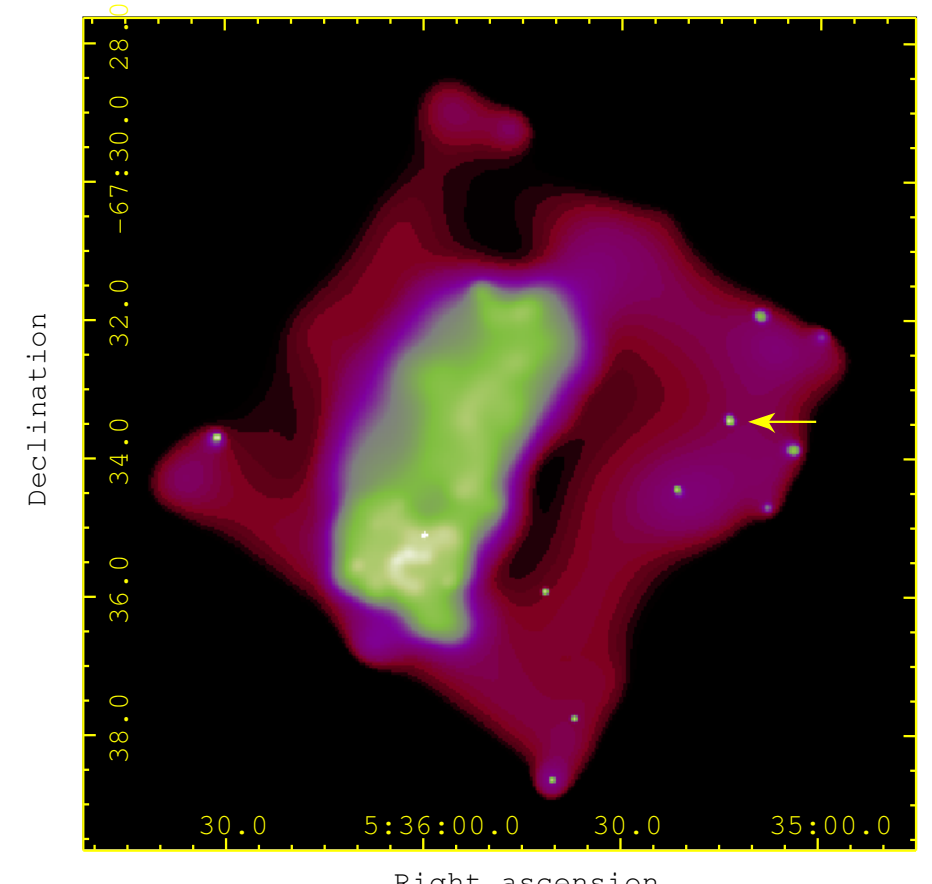

Fig. 2.- The field of the $8^{\prime} \times 8^{\prime}$ Chandra S3 chip. This is an adaptive smoothing of X-ray data in the energy range 0.3 - $3 \mathrm{keV}$. The color map shows increasing surface brightness going from red to green to white. The scale has been set so that the supernova remnant in this figure is green. The point source in the SE part of the remnant is quite bright and is unresolved by Chandra. The next Figure better shows the true prominence of this source. Other unresolved sources in this field are foreground or background objects. One of these, the star HD 269810, is indicated by an arrow and serves as a fiducial reference. 


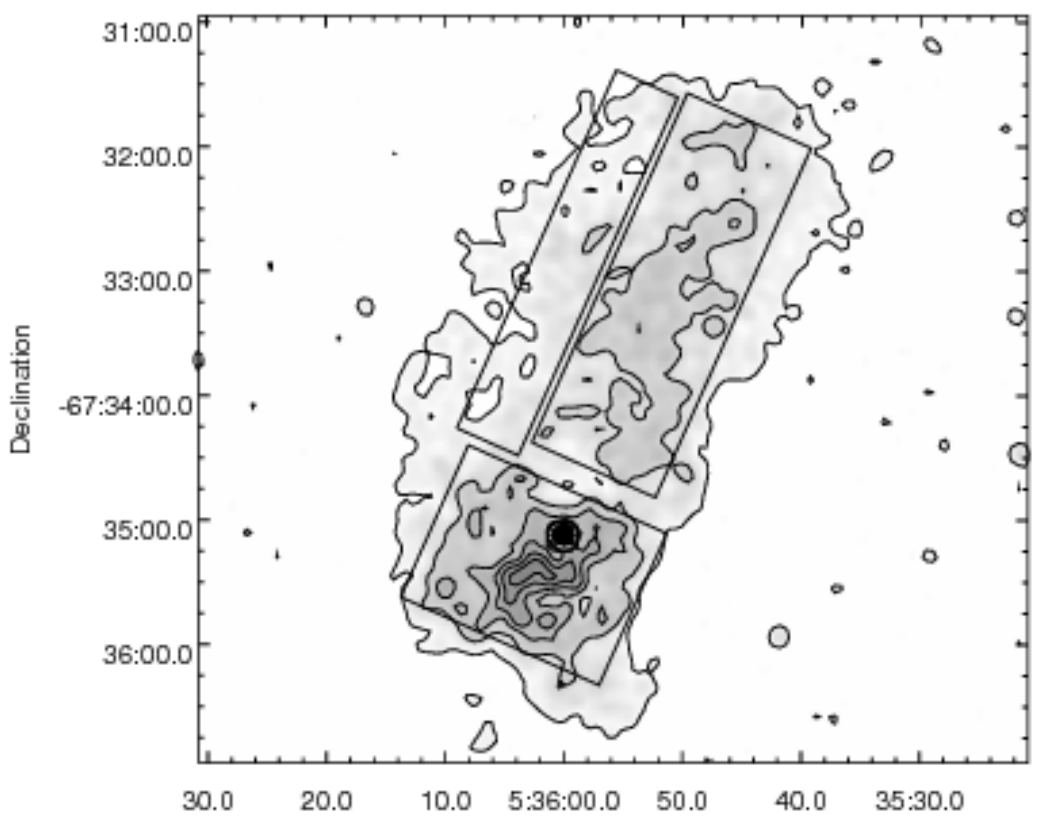

Right ascension

Fig. 3.- Chandra observation of the remnant showing contours of constant X-ray surface brightness overlaid on a smoothed image. Smoothing is Gaussian with $\sigma=5^{\prime \prime}$. Contours are linear with separation 0.3 counts arcsecond ${ }^{-2}$. Note the diffuse structure just south of the bright unresolved source. The three boxes show spectral extraction regions. 


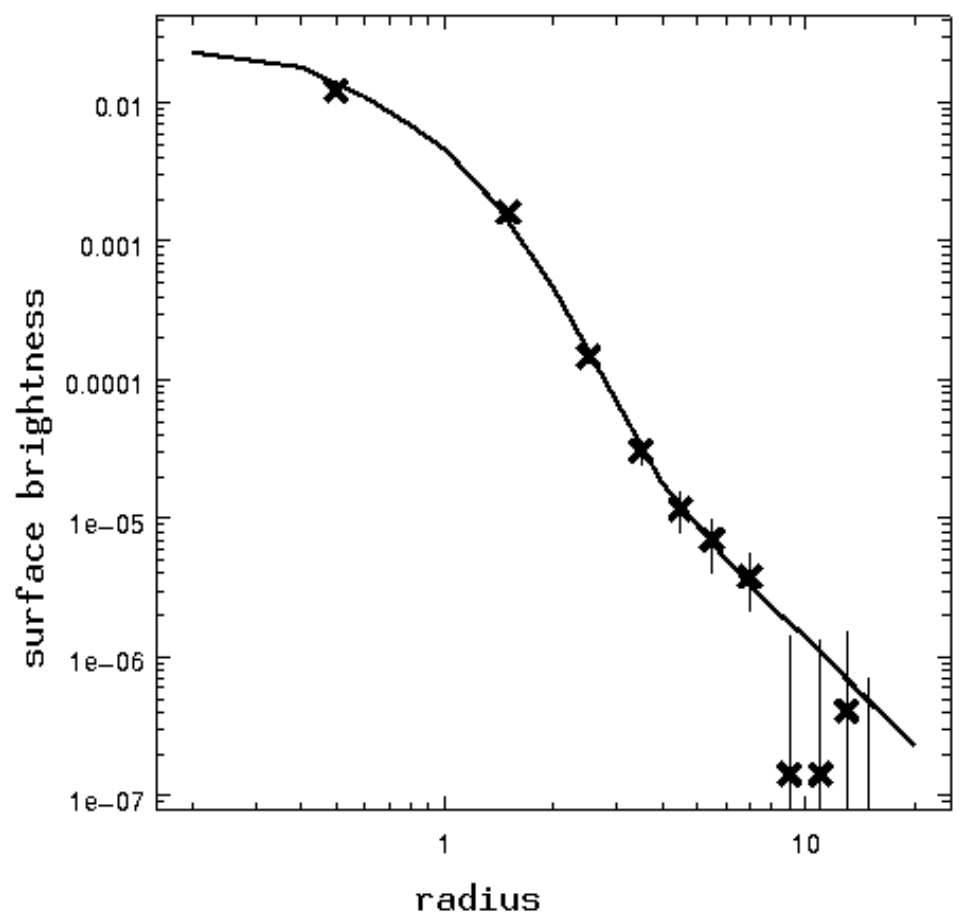

Fig. 4.- Radial surface brightness of CXOU 053600.0-673507 compared with the Chandra telescope point spread function (PSF). The diffuse SNR emission has been subtracted. Data points are crosses with $1 \sigma$ uncertainties due to counting-statistics. The solid curve is the Chandra mirror PSF from Jarius 2002. Units of radius are ACIS pixels (=0.492 arcsecond). Units of surface brightness are counts $s^{-1}$ pixel $^{-1}$. 
jdpileup, in the Chandra CIAO/SHERPA spectral analysis. Results are listed in Table 1. The best fit was a power law with index $-1.28 \pm 0.08$ and is shown in the figure. Without the pileup correction the best-fit index was $-1.10 \pm 0.06$. (The uncertainties given are $1 \sigma$. ) The index measured with XMM, free of pile up but with larger background subtraction, was $-1.57 \pm 0.05$ (Bamba et al 2006). The lack of agreement could be due to an inadequate pileup correction, larger uncertainties than quoted, or a variable source. The Chandra and XMM-measured luminosities are the same. The luminosity range in Table 1 is due to the observed variability. We also fit a $x s d i s k b b$ model to the data since this is appropriate for accretion powered sources and there is reason to believe this object may be part of a binary system. The fit is reasonable but the absorption is too low. If this is an accretion powered system, a more elaborate model is needed.

Table 1. Compact source spectral fits

\begin{tabular}{llllll}
\hline \hline form & $\begin{array}{l}\text { energy range } \\
(\mathrm{keV})\end{array}$ & photon index & $\begin{array}{l}\text { ISM absorption } \\
N_{H}\left(10^{22}\right)\end{array}$ & $\begin{array}{l}\text { reduced } \\
\chi^{2}\end{array}$ & $\begin{array}{l}L_{x} \\
\left(\mathrm{erg} \mathrm{s}^{-1}\right)\end{array}$ \\
\hline power law & $0.3-10$ & $\Gamma=1.28 \pm 0.08$ & $0.19 \pm 0.034$ & 1.26 & $2.5-3.2 \times 10^{35}$ \\
diskbb & $0.3-10$ & $k T_{i n}=2.43 \pm 0.23$ & $0.073 \pm 0.023$ & 1.38 & $2.3 \times 10^{35}$ \\
\hline
\end{tabular}

\subsection{Location and variability}

The bright optical counterpart, a $\mathrm{V}=13.5 \mathrm{O} 5 \mathrm{III}(\mathrm{f})$ star, is easily visible within the Head of the remnant in Figure 1. This had been noted as a possible counterpart to the Einstein source CAL 60 (from the catalog of Long et al, 1981) by Crampton et al (1985) who published a finding chart and spectrum. Since the source CAL 60 includes the diffuse emission as well as the point source we will refer to the point source as CXOU 053600.0-673507. Our X-ray position is $2.2^{\prime \prime} \mathrm{S}$ of the XMM position and $<0.6^{\prime \prime}$ from this $\mathrm{O}$ star. X-ray positions are listed in Table 2 with uncertainty the larger of that from counting statistics or the difference between positions from the two halves of our observation. The 


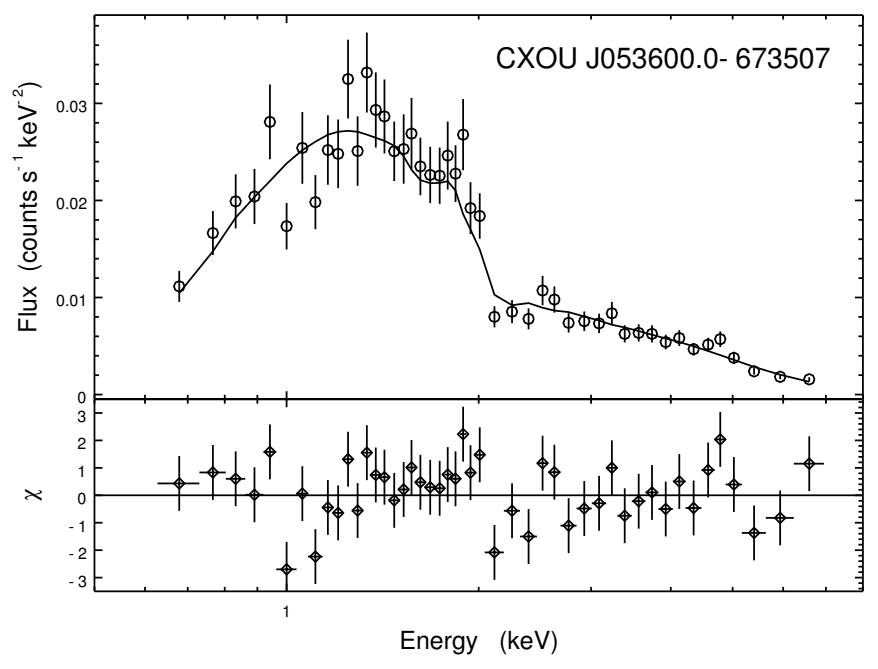

Fig. 5.- X-ray spectrum of the compact source. The solid line is the best-fit power law with pileup correction and the lower panel illustrates deviation of data points from this fit. Uncertainties are $1 \sigma$ in number of counts.

systematic error associated with Chandra positions is $0.6^{\prime \prime}$ at $90 \%$ confidence. The star HD2269810 is also in the field and our X-ray position for this star is $0.5^{\prime \prime}$ from the optical and 2MASS IR survey position, so the registration of the Chandra field is good and we adopt $0.6^{\prime \prime}$ as the uncertainty of the X-ray position.

The source is variable. The count rate in the second part of the observation increased $25 \%$ over that obtained in the first part. No variability was seen on time scales shorter than

Table 2. Measured source positions

\begin{tabular}{llll}
\hline \hline source & waveband & RA $(2000)$ & Dec $(2000)$ \\
\hline CXOU J053600.0-673507 & X-ray & $05: 36: 00.01 \pm 0: 0: 0.02$ & $-67: 35: 07.5 \pm 0: 0: 0.2$ \\
O star & optical & $053559.9 \pm 0: 0: 0.08$ & $-673506.3 \pm 0: 0: 0.5$ \\
O star & IR & $05: 36: 00.01$ & $-67: 35: 07.6$ \\
\hline HD 269810 & X-ray & $05: 35: 13.82 \pm 0: 0: 0.05$ & $-67: 33: 28.0 \pm 0: 0: 0.3$ \\
HD 269810 & IR & $05: 35: 13.89$ & $-67: 33: 27.6$ \\
HD 269810 & TYCHO-2 & $05: 35: 13.90$ & $-67: 33: 27.6$ \\
\hline
\end{tabular}


$\sim 10^{4} \mathrm{~s}$ although the observation was not sensitive to periods shorter than $6 \mathrm{~s}$ or to pulsed fractions $<20 \%$. This object cannot be an unresolved PWN as was reasonably inferred from the XMM observation by Bamba et al.

Since in $\approx 12$ hours the Chandra flux varied $25 \%$ we searched for variability in past observations. These were all of limited sensitivity but could show variability extremes. Table 3 lists imaging observations which have detected this remnant. We inspected the archival Einstein and ROSAT fields and, in all cases could distinguish the Head and Tail of the remnant but could not separate the point source in the Head from the diffuse emission. The diffuse and compact parts of the remnant are only well separated in XMM and Chandra observations. Indeed, the spatial resolution and low count rate in Einstein and ROSAT observations make it impossible to do this if the point source is no stronger than in our observation. In these past observations only $10-20 \%$ of the counts in the Head should be from the point source and total counts from the Head range from $\sim 30$ to $~ 200$ with the higher rates from detectors with the lower spatial resolution. In all cases the counting rate was about the same from the Head and Tail regions as it is in the Chandra observation, so this is reassuring.

In order to compare observations of the diffuse source we have converted count rates

Table 3. X-ray observations of DEM L241

\begin{tabular}{|c|c|c|c|c|c|c|}
\hline date & spacecraft & energy range & $\begin{array}{c}\text { compact source } \\
0.5-5 \mathrm{keV} \text { abs. flux } \\
\left(10^{-13} \mathrm{erg} \mathrm{cm}^{-2} \mathrm{~s}^{-1}\right)\end{array}$ & $\begin{array}{l}\text { compact source } \\
L_{x} 0.3-10 \mathrm{keV} \\
\left(10^{35} \mathrm{erg} \mathrm{s}^{-1}\right)\end{array}$ & $\begin{array}{c}\text { diffuse absorbed } \\
0.5-5 \mathrm{keV} \text { Head flux } \\
\left(10^{-13} \mathrm{erg} \mathrm{cm}^{-2} \mathrm{~s}^{-1}\right)\end{array}$ & reference \\
\hline 1979 Apr 10 & Einstein IPC & $0.15-4$ & - & - & $4.1 \pm 1.4$ & Long et al 1981 \\
\hline 1979 Nov 5 & Einstein HRI & $0.15-4.5$ & - & - & $5.4 \pm 1.4$ & Mathewson et al 1985 \\
\hline 1991 Feb 14 & ROSAT HRI & $0.15-2.5$ & - & - & $6.0 \pm 1.6$ & Schmidtke et al 1994 \\
\hline 1993 Jul 24 & ROSAT PSPC & $0.15-2.5$ & - & - & $4.2 \pm 0.8$ & \\
\hline 1995 Jul 9,17 & ROSAT HRI & $0.15-2.5$ & - & - & $5.1 \pm 1.0$ & Schmidtke et al 1999 \\
\hline 2004 Dec 29 & $\mathrm{XMM}$ & $0.5-10$ & & $2.32 \pm 0.14$ & & Bamba et al 2006 \\
\hline 2011 Feb 7 & Chandra & $0.3-10$ & $3.71 \pm 0.10$ & $2.52 \pm 0.07$ & $4.42 \pm 0.12$ & this paper \\
\hline $2011 \mathrm{Feb} 8$ & Chandra & $0.3-10$ & $4.70 \pm 0.12$ & $3.19 \pm 0.08$ & $4.43 \pm 0.12$ & this paper \\
\hline
\end{tabular}


to flux levels with PIMMS, assuming a thermal spectrum with $k T=0.5 \mathrm{keV}$ and $N_{H}$ $=2 \times 10^{21} \mathrm{~cm}^{-2}$. To avoid large absorption corrections at energies below $0.5 \mathrm{keV}$, we calculated the flux in the range 0.5 to $5.0 \mathrm{keV}$ and these results are summarized in Table 3. Uncertainties are counting statistics only. The luminosity, $L_{X}$, of the compact source is calculated for the Chandra range $0.3-10 \mathrm{keV}$. The agreement of past observations is good, as indeed it should be for the diffuse emission. Any variation of the point source less than a factor of $\sim 3$ is undetectable in the Einstein and ROSAT data. The past observations of this remnant therefore show that there have been no major outbursts at these times.

We searched for regular pulsations in the Chandra data and found nothing significant. Since the ACIS instrument integrates for $3.2 \mathrm{~s}$, the search was only valid for periods between $6.4 \mathrm{~s}$ and $6 \mathrm{~h}$ and the signal of 2500 counts limited the search to pulsed fraction above $\approx$ 20\%. Bamba et al. searched XMM and ASCA data in the period range $0.15-500 \mathrm{~s}$ at about this same sensitivity with null result. A short period and/or a $\approx 10 \%$ pulse fraction is quite possible.

\subsection{Optical/NIR}

We have measured the optical spectrum of this star with the SAAO $1.9 \mathrm{~m}$ telescope at Sutherland, South Africa in October 2011 and April 2012. The spectrum from 2011 Oct 24 is shown in Figure 6. This spectrum is almost identical to that of Crampton et al, indicating no long-term spectral variability. The velocity we measure from the Balmer (mean of $\mathrm{H}-\beta$ and $\mathrm{H}-\gamma$ ) absorption lines is $324 \pm 7 \mathrm{~km} \mathrm{~s}^{-1}$ so it is clearly in the LMC and an association with the remnant is possible. Further spectra on 2012 April 12 and 15 yielded velocities of 301 and $296 \mathrm{~km} \mathrm{~s}^{-1}$ respectively (and with similar uncertainties). These values are consistent with the Crampton et al (1985) mean value of $304 \mathrm{~km} \mathrm{~s}^{-1}$, as well as the

variability by $34 \mathrm{~km} \mathrm{~s}^{-1}$ that was noted then. The small velocity change noted between our 


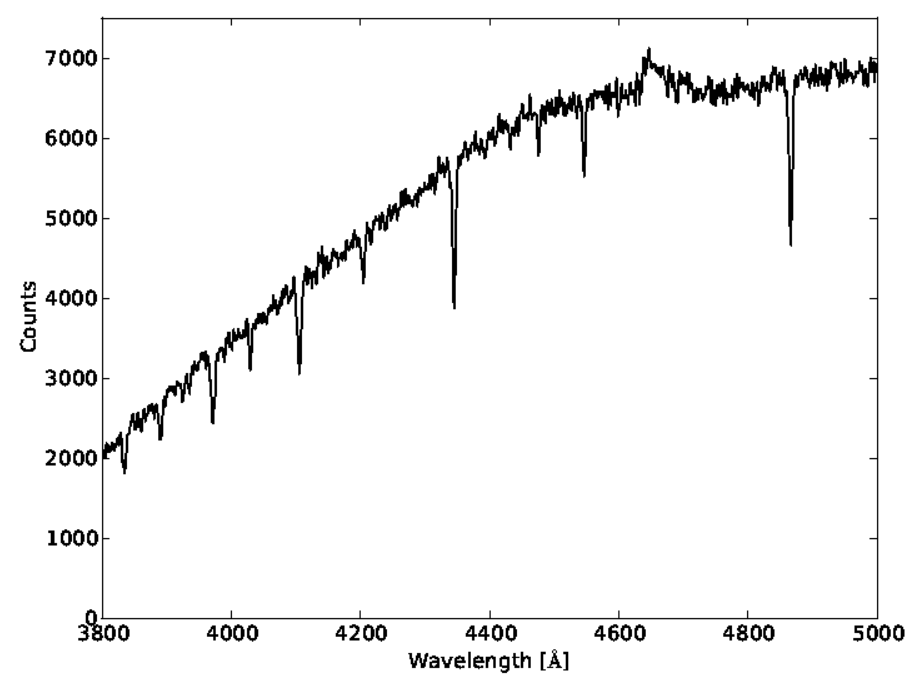

Fig. 6. - Spectrum of the O5III(f) counterpart of CXOU 053600.0-673507 taken with the SAAO 1.9m telescope. Absorption lines are from $H$ and $H e$. The emission feature is the Bowen blend, typical of very early $O$ stars.

April spectra are indicative of a long period system, likely tens of days or more.

IR photometry in the $\mathrm{J}, \mathrm{H}$ and $\mathrm{K}_{S}$ wavebands of the optical counterpart was obtained during the month of November 2011 using the 1.4m IRSF telescope at SAAO's Sutherland observing station. These showed no significant variability to within 0.1 mag.

\section{The Diffuse Emission}

\subsection{Radio Observations}

Moderate-resolution radio observations of this area were made during surveys at 8.6and 4.8-GHz (Dickel et al. 2005), at $1.4 \mathrm{GHz}$ (Hughes et al. 2007), and at $843 \mathrm{MHz}$ (Mauch et al. 2003). The best resolution is at $8.6 \mathrm{GHz}$ with a half-power beam width of 
20". An overlay of the X-ray image on the $8.6 \mathrm{GHz}$ radio contours is shown in Fig. 7. The X-ray emission has been smoothed with a 5-arcsecond Gaussian. The H II region is clearly delineated by the contours with its two brightest parts N59A on the west and N59B on the east as designated by Henize (1956). Because of the overlap with the bright H II region it is difficult to separate a possible contribution of the SNR to the radio emission. The rms noise should give a 5 sigma limit of about $1 \mathrm{mJy} /$ beam but the contamination could make the limit a few mJy/beam. The fact that no radio emission appears to match with the X-ray emission is unusual. Most SNRs with significant X-ray emission are readily visible at radio wavelengths as well. Apparently the surrounding H II region complex masks the non-thermal emission from the SNR.

With the current resolution, any point radio source would be difficult to distinguish from the somewhat irregular background, but the absence of any feature at the position of the point source suggests that it would be less than a few mJy. For comparison, the X-ray only pulsar 0537-6910 in N157B is less than .06 mJy at $22 \mathrm{~cm}$ (Crawford et al. 1998) and PSR 0540-693 in the composite SNR of the same name is $0.4 \mathrm{mJy}$ at $640 \mathrm{MHz}$. N206 is a shell-like radio SNR with a radio jet-like feature with an X-ray point source at the tip, but no radio point source or pulsar at $<.02 \mathrm{mJy}$ (Williams et al. 2005). Thus the radio limit on the point X-ray source is not very significant or unexpected.

Another way to distinguish between the thermal emission from $\mathrm{H}$ II regions and the non-thermal synchrotron emission from SNRs is the radio spectrum. H II regions have a nearly flat spectrum with a slope, or spectral index, near 0 while PWN have a modest decrease in brightness as frequency increases with an index of about -0.2 to -0.3 . Shell SNRs have average spectral index slopes of -0.5 with young remnants being steeper and old ones flatter. Figure 8 shows a four-wavelength radio spectral index map of the entire region. Production of this image required convolution of all the data to the $48.5 \times 45.0$ 


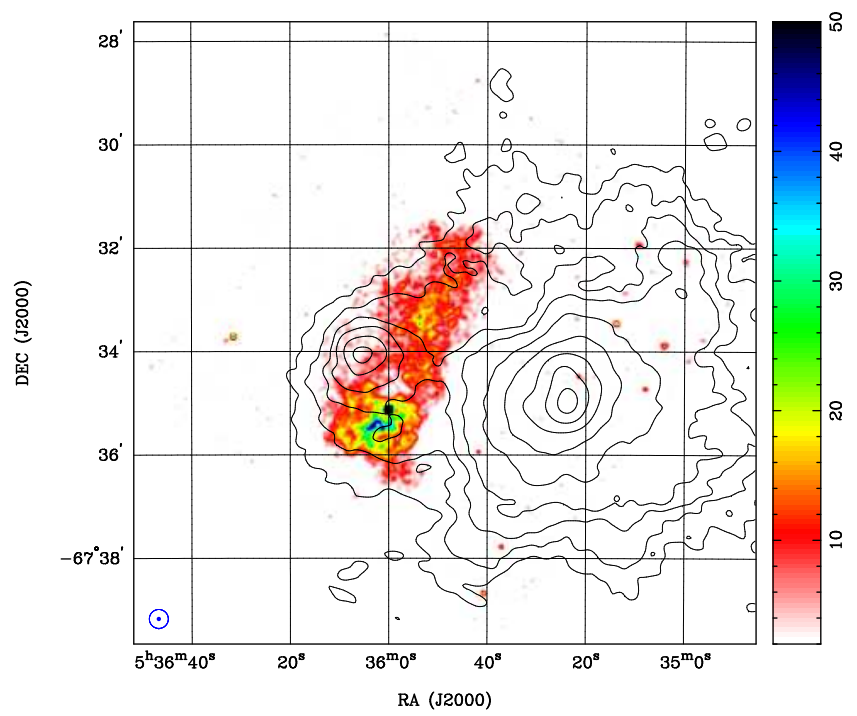

Fig. 7.- Contours of constant $3.5 \mathrm{~cm}$ radio surface brightness overlaid over the smoothed X-ray emission. The X-ray field is that of Figure 2 but with $5^{\prime \prime}$ Gaussian smoothing. Radio contours are drawn at 2, 3, 4, 5, 7, 10, 20, $50 \& 100 \mathrm{mJy} /$ beam. The dot in the lower left represents the 5-arcsec resolution of the smoothed X-ray image and the surrounding circle is the 20-arcsec beam width at $3.5 \mathrm{~cm}$. The two radio maxima are from the bright $H$ II regions seen in Figure 1. 


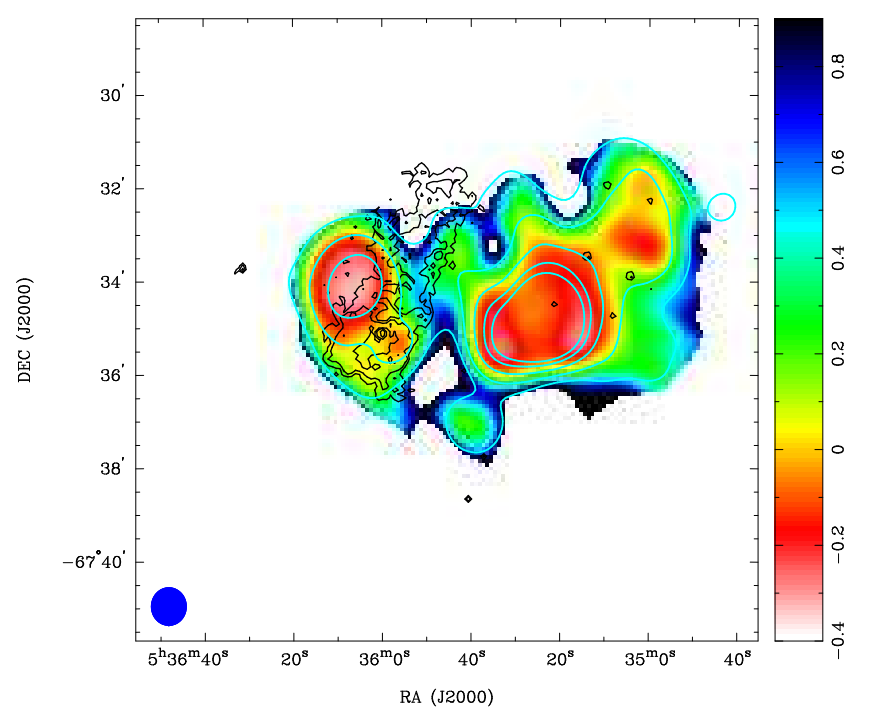

Fig. 8.- Color indicates the radio spectral index with red showing the flat spectrum characteristic of thermal emission. There is no indication of the steeper spectrum expected from the shell of a supernova remnant. Smoothed contours of constant $21.7 \mathrm{~cm}$ radio surface brightness are overlaid and drawn at 10, 20, 40, 70, \& $100 \mathrm{mJy} /$ beam where the 48.5"x45" radio beam is indicated at the bottom left of the figure. Black contours show the X-ray surface brightness at levels of 0.25, 0.5, 1, 2.5, and 12 counts arcsecond ${ }^{-2}$. 
arcsec resolution of the 36 -cm image. Obviously, part of the X-ray Tail is missing in the radio but other areas could contain some synchrotron emission from a PWN or very old SNR in addition to the thermal emission from the H II regions. From the spectral index map, we cannot distinguish the separation of various components.

\subsection{Optical Observations}

For comparison with optical wavelengths, we used images from the Magellanic Cloud Emission Line Survey (MCELS; Smith 1999). These images were taken at the UM/CTIO Curtis Schmidt telescope at Cerro Tololo Inter-American Observatory (CTIO). The detector, a Tek 2048 x 2048 CCD with 24 micron pixels, gave a scale of 2.3 arcsec per pixel and a resulting angular resolution of approximately 2.6 arcsec. The narrow band images were taken with filters centered on the [O III] (5007, FWHM $=40 \AA)$, H-alpha $(6563 \AA, F W H M=30 \AA)$, and $[\mathrm{S} \mathrm{II}](6724 \AA$, FWHM $=50 \AA)$ emission lines. The image shown in Figure 1 has been reduced using the IRAF software package for bias subtraction and flat-field correction, and the astrometry was derived based on the HST Guide Star and USNO-A catalogs. This image, however, has not been flux-calibrated or continuum-subtracted.

Because of the presence of the bright H II region, a good way to depict the SNR is to show the $[\mathrm{S} \mathrm{II}] / \mathrm{H} \alpha$ ratio. The $[\mathrm{S} \mathrm{II}]$ doublet at 671.7 and $673.1 \mathrm{~nm}$ is enhanced by

shocked material so any region with an $[\mathrm{S} \mathrm{II}] / \mathrm{H} \alpha$ ratio greater than about 0.5 is considered a supernova remnant. In Fig. 9 we show the ratio superimposed on contours of the X-ray emission. There is clearly a good correlation except on the eastern edge where the bright $\mathrm{H}$ II-region component N59B overwhelms emission from the remnant.

No X-rays are observed from the outer shock traced by the [S II] filaments. This implies 
that the temperature of hot gas in the shock is $<8 \times 10^{5} \mathrm{~K}$. The Sedov model then, can be used to estimate an age limit. Assuming $E_{0}=10^{51} \mathrm{ergs}$ and using the densities given in Table 5 , we derive an age of $>5-7 \times 10^{4}$ years.

\subsection{X-rays}

There are two major parts to this object. The X-ray spectra of the Head and the Tail are sufficiently similar to indicate that they are parts of the same remnant rather than two separate objects and we will so treat them. Data were extracted from the 3 regions outlined in Figure 3 - the Head or S region, the bright NW strip which accounts for $80 \%$ of the emission from the Tail and a NE strip where there is fainter emission which might be indicative of a shock. After background subtraction the number of events in these regions was: S - 7960, NW - 4640 and NE - 1020. The first two regions contain enough events to support an interesting spectral analysis but the faint NE region yields less information. Background was taken from large source-free regions northeast and southwest of the remnant and was $35 \%$ of the remnant signal in the S region, $85 \%$ of the NW signal and $200 \%$ of the NE signal. The spectra of these regions are shown in Fig 10. The visually distinct spectra of the S and NW regions can be explained almost entirely by differences in absorption of the emitted X-rays. This can be seen by inspection of the softest emission and is greatest in the region between Head and Tail. The visual gap in the X-ray image which separates the Head from the Tail is most prominent in the softest energy band and is caused by absorbing material. This is not surprising considering the complex surrounding environment. The spectra of the bright structure just south of the $\mathrm{O}$ star and the surrounding outer part of the Head were examined separately and were, within uncertainties, the same. The bright structure is therefore plausibly explained as a density enhancement within the SNR or an extension of the remnant structure along the line of 


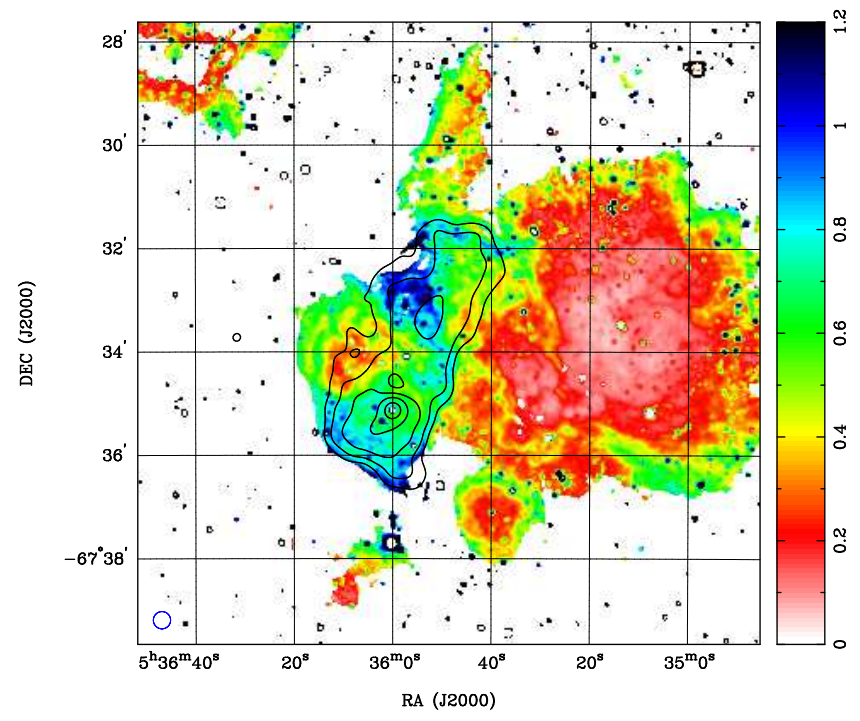

Fig. 9.- Contours of constant X-ray surface brightness overlaid on the ratio of [S II] to Balmer optical emission. X-ray data have been smoothed with a 20" Gaussian and X-ray contours are drawn at levels of 0.12, 0.25, 0.5, $1 \& 2$ counts arcsecond ${ }^{-2}$. 
sight.

We used the xsvpshock model, which accounts for non-equilibrium ionization in a one-dimensional shock, to fit the NW region which shows least absorption. Metal abundances were fixed at 0.3 solar and then allowed to vary to fit the data. It was necessary to vary only $\mathrm{O}, \mathrm{Ne}, \mathrm{Mg}$, and Fe. We then fit the $\mathrm{S}$ and $\mathrm{NE}$ regions with the same spectrum allowing only the ISM absorption and timescale parameters to vary. Best-fit parameters are listed in Table 4. The NW fit is excellent, The S fit is good and the NE fit as good as any that can be achieved with the limited number of events. These fits are shown in Figure 10 . The difference between fits of the S and NW regions is entirely due to different absorption of the $\mathrm{O}, \mathrm{Ne}$ and Fe emission lines. The fit to the NE region requires a lesser value of the ionization timescale $\left(\tau_{\mu}\right)$. Since the NE region comprises the outer part of the expanding remnant, this is expected.

The formal $1 \sigma$ uncertainties in the S and NW spectral parameters due to counting statistics are $\approx 15 \%$ for the absorption $\left(N_{H}\right),<10 \%$ for the temperature $(k T), \sim 40 \%$ for the abundances and $\sim 50 \%$ for the ionization timescale $\left(\tau_{\mu}\right)$. The statistical uncertainties of the data points, however, allow acceptable fits over a large range of parameters, e.g. the temperature can be varied by $\pm 50 \%$ and reasonable fits can be obtained with small changes in abundances (although the Fe abundance is more sensitive than the lighter elements). Table 4 lists CIAO/SHERPA best-fit spectral parameters for the xsvpshock and the equilibrium xsvmekal models. 
Table 4. Diffuse source spectral fits

\begin{tabular}{l|ccl|lc}
\hline \hline region & NW Tail & S Head & NE Tail & NW Tail & S Head \\
\hline model & \multicolumn{3}{|c|}{ xsvpshock } & \multicolumn{2}{|c}{ xsvmekal } \\
\hline reduced $\chi^{2}$ & 0.79 & 1.05 & 1.38 & 0.91 & 1.08 \\
$N_{H}$ & 0.14 & 0.37 & 0.17 & 0.22 & 0.29 \\
\hline $\mathrm{kT}$ & & 0.52 & & 0.33 & 0.41 \\
$\mathrm{O}$ & & 1.9 & & 1.2 & 2.9 \\
$\mathrm{Ne}$ & & 3.2 & 1.3 & 4.9 \\
$\mathrm{Mg}$ & & 1.7 & & 1.0 & 2.3 \\
$\mathrm{Fe}$ & & 0.4 & & 0.2 & 0.35 \\
\hline$\tau_{\mu}$ & $7 \times 10^{11}$ & $7 \times 10^{11}$ & $2 \times 10^{11}$ & - & - \\
\hline
\end{tabular}

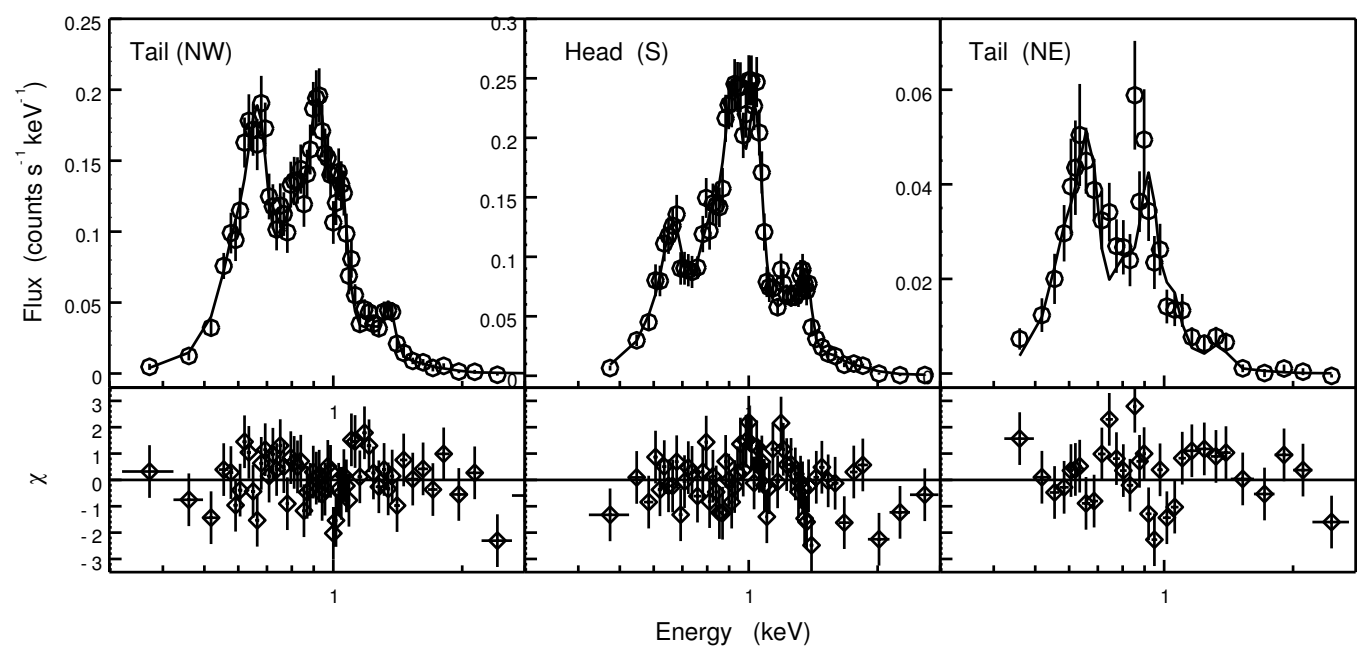

Fig. 10.-X-ray spectrum of the three diffuse regions. The lower panel illustrates deviation of data points from the best fit xsvpshock model shown by the solid lines. A linear scale is used to show the large apparent differences between spectra from the three regions. Only the absorption and timescale parameters vary from region to region. Prominent emission lines are $\mathrm{O}$ at $0.67 \mathrm{keV}, \mathrm{Ne}$ and $\mathrm{Fe}$ at $\approx 1 \mathrm{kev}$, and $\mathrm{Mg}$ at $1.32 \mathrm{keV}$. 


\section{Discussion}

\subsection{Nature of compact object}

The variability excludes an unresolved PWN such as that surrounding the Vela Pulsar. Furthermore the Vela PWN has $L_{x}=2 \times 10^{33} \operatorname{ergs~s}^{-1}$, considerably less than observed here. The compact object is too bright to be a Cas A-type CCO (Central Compact Object with $L_{x}=10^{33}-10^{34}$ ergs s$^{-1}$ ). The luminosity is compatible with an AXP (Anomalous X-ray Pulsar with $L_{x}=10^{35}-10^{36} \operatorname{ergs~s}^{-1}$ ) but the spectrum is harder than that of most AXP (McGill Pulsar Group 2012). The hard spectrum suggests an accretion-powered binary which, comprising an O star and a neutron star, would be a High-Mass X-ray Binary (HMXB). This is unexpected. Only two other X-ray binaries have been discovered within observable SNR - SS 433, with a B or A giant primary star, within

the galactic remnant W50 and recently, a Be X-ray pulsar system, SXP 1062, within a faint Small-Magellanic-Cloud remnant (Hénault-Brunet 2011).

Although we have no evidence other than location that the $\mathrm{O}$ star under discussion and the X-ray source form a binary system, we suspect that the object within DEM L241 is the third HMXB to be found within a SNR. The compact object, in this HMXB, if a neutron star, would accrete material from the O-star wind and changes in luminosity would be caused by changes in the density of the wind. Scaling from the HMXB Cen X-3, the moderate luminosity and lack of observed outbursts imply (very roughly) a separation of $\sim$ $1 \mathrm{AU}$ and an orbital period of weeks. At the moment this is speculation but the velocity of the O-star has indeed been observed to vary. No regular pulsation in the X-rays have been found but the observations were not sensitive to pulsed fractions below $\sim 20 \%$.

The fact that the supernova precursor must have been more massive than the O-star companion implies a precursor mass of 25 , perhaps $40 M_{\odot}$, thought to be the mass necessary 
for collapse to a black hole. It is therefore possible that the compact object, which we have assumed to be a neutron star, could actually be a black hole. The X-ray luminosity, however, is unusual for a black hole. Known black hole binaries are either bright with $L_{x} \geq 10^{37} \mathrm{erg} \mathrm{s}^{-1}$ or are transient systems in quiescence with $L_{X}<10^{33} \mathrm{erg} \mathrm{s}^{-1}$ (Remillard and McClintock 2006).

However, the HMXB in DEM L241 has a luminosity (2/times10 $\left.{ }^{35} \mathrm{erg} \mathrm{s}^{-1}\right)$, intermediate between these extremes. Interestingly, this is similar to the luminosity of SS433. While there is as yet no direct dynamical measurement of the compact object mass in SS433, all the indirect evidence strongly supports it being a $\sim 15 M_{\odot}$ black hole accreting at extremely high mass transfer rates from a highly evolved supergiant primary (see e.g. Blundell et al 2008). The reason that it's X-ray luminosity is so low $\left(\sim 10^{35} \mathrm{erg} \mathrm{s}^{-1}\right)$ is that it is observed at high inclination (it is eclipsing), and hence we only receive X-rays scattered from the surrounding material into our line of sight. i.e. it is an "accretion disc corona" system.

As discussed earlier, there is marginal evidence that $L_{x}$ of the HMXB in DEM L241 does not vary much, but we consider it unlikely that it too would be a high inclination system. Consequently, a black hole is certainly possible but we have no strong indication that this might be the case. A measure of the velocity variation of the O5III(f) companion would provide a test of this hypothesis. This remnant could become the first example of a remnant known to be created by stellar collapse to a black hole.

\subsection{Nature of the remnant}

Throughout this discussion it is assumed that the precursor star was originally bound to the O5III(f) star. After the SN, the compact remnant of this remained bound to the O star to form the present HMXB. The explosion itself probably determined the size and 
temperature of the remnant. The binary environment of the precursor may have shaped the remnant morphology.

The X-ray morphology of the SNR looks like that of a PWN with an extended elliptical structure showing no obvious shell and a point X-ray source near one end. However, all diffuse emission, including that close to the point source, is well fit with a thermal spectrum. There is no evidence for a power law component although a $10 \%$ contribution cannot be excluded. The X-ray extent along the major axis of about 80 pc makes the object one of the larger of the known SNR. The SN may have exploded between the two major H II regions and the expansion was impeded in the east-west direction. However, although the SNR and the H II region complex do appear coincident in the sky there is no indication in their structures that they have interacted so they could be at different distances along the line of sight.

The vpshock fits indicate an age of $\tau_{\mu} / n_{e} \sim 7 \times 10^{11} / 0.14-0.27 \mathrm{~s}=0.8-1.6 \times 10^{5} \mathrm{yr}$, about twice the lower limit estimated using the Sedov model. This age is old for a radio SNR as the expanding shock will have slowed sufficiently to reduce the shock acceleration of relativistic particles that create the radio synchrotron emission. Thus the age can explain both the relatively flat radio spectral index and the weak radio emission in general.

Since the plasma is close to equilibrium we also fit the X-ray spectra with the xsvmekal equilibrium model. Table 4 gives the best fit result of this model to the S and NW spectra. In this case, the two spectra have been fit separately. The S region, the Head surrounding the compact object requires somewhat higher temperature and abundances than the NW Tail region. Absorption is still higher in the S than in the NW region.

Although there is considerable uncertainty in the spectral fit parameters and in the volumes used for the three regions, enhanced abundances and derived densities indicate that we are seeing radiation from supernova debris above that from the normal ISM in 
the Magellanic Clouds. Table 5 gives the plasma densities and masses calculated from the vpshock results of Table 4. The excess of $\mathrm{O}, \mathrm{Ne}$, and $\mathrm{Mg}$ above that expected is 2.5, 0.8, and $0.15 M_{\odot}$, indicating the explosion of a massive star. Woosley and Weaver (1995) have calculated the masses of elements ejected from explosions of massive stars. This amount of O can come from a precursor with mass $>25 M_{\odot}$ and the Ne from a precursor with mass $>40 M_{\odot}$. Since there is variation in the different Woosley \& Weaver models and uncertainty in our measured abundances, we can only conclude that the precursor was massive. Since it evolved first, it should have been more massive than the present O5III(f) star, so this is expected. Martins et al. (2005) give the mass of an isolated O5III(f) star as about $40 M_{\odot}$. However, Rappaport \& Joss (1983) show evidence that the companion stars in HMXBs are "undermassive for their luminosity", so a precursor mass estimate of $>25 M_{\odot}$ is more conservative.

\section{Table 5. Properties of the remnant}

\begin{tabular}{l|cccccc}
\hline \hline region & $F_{x}$ & $L_{X}$ & Volume & $n_{e}$ & Mass & $E_{t h}$ \\
\hline & $\mathrm{ergs} \mathrm{cm}^{-2} \mathrm{~s}^{-1}$ & $\mathrm{ergs} \mathrm{s}^{-1}$ & $\mathrm{~cm}^{3}$ & $\mathrm{~cm}^{-3}$ & $M_{\odot}$ & $\mathrm{ergs}$ \\
\hline S Head & $4.17 \times 10^{-13}$ & $6.10 \times 10^{35}$ & $2.8 \times 10^{59}$ & 0.27 & 83 & $1.9 \times 10^{50}$ \\
NW Tail & $3.86 \times 10^{-13}$ & $2.36 \times 10^{35}$ & $4.1 \times 10^{59}$ & 0.14 & 61 & $1.1 \times 10^{50}$ \\
NE Tail & $0.83 \times 10^{-13}$ & $0.68 \times 10^{35}$ & $1.6 \times 10^{59}$ & 0.12 & 21 & $0.4 \times 10^{50}$ \\
Total SNR & - & $9.14 \times 10^{35}$ & $8.5 \times 10^{59}$ & - & 165 & $3.4 \times 10^{50}$ \\
\hline
\end{tabular}

The remnant parameters derived here differ somewhat from those obtained from the XMM observation by Bamba et al (2006). Our X-ray flux and luminosity is $30 \%$ higher because we use a different energy band. Our total mass is $30 \%$ lower because we assume different volumes for the emitting regions. Bamba et al assume ellipsoidal volumes for Head and Tail whereas we use cylindrical volumes with different dimensions. The Chandra best-fit spectra have higher temperature and greater enrichment. Remarkably, the total thermal energy content is the same for the two analyses.

The elongated structure may be due to a "blowout" to a lower density region in the 
north. If so, this was a major event. The Tail now has twice the volume of the Head and contains $40 \%$ of the thermal energy of the hot gas. On the other hand, perhaps the unusual elongation of the remnant is associated with the unusual central source which is quite likely a HMXB. The massive binary might have produced a disk of material which channeled the ejecta into two lobes. Or, the precursor star itself could have been a Wolf-Rayet star with strong wind which shaped a cavity now filled by the remnant. Or, rotation of the precursor could have produced an asymmetrical explosion.

\subsection{Comparison with W50/SS433}

There is an interesting parallel with the morphology of the Galactic remnant W50 which is also large and elongated with dimension $95 \times 200 \mathrm{pc}$ (at a distance of $5.5 \mathrm{kpc}$, Lockman et al 2007). The binary SS 433 lies at the center of W50 and produces opposing relativistic jets which have inflated and elongated the remnant in the EW direction. The optical manifestation of these jets is spectacular (Margon et al 1980). SS 433 is an unresolved X-ray source with luminosity $3 \times 10^{35} \mathrm{ergs} \mathrm{s}^{-1}$, the same as that of the DEM L241 source.

DEM L241, with dimension $32 \times 77 \mathrm{pc}$, is also elongated but only about one third the size of W50. From the O star to the northern end of DEM L241 is 57 pc, about half the length of $120 \mathrm{pc}$ for the longer arm of W50. Perhaps the Tail of DEM L241 was inflated by a single northern jet and the structure south of the O star is due to energy deposited by the oppositely-directed jet. Since we see no other evidence for jets, this probably happened in the past and is no longer operating. The lesser size of DEM L241 also indicates a weaker jet and/or a younger remnant.

Despite intensive searches, no other examples of SS 433/W50 systems have been found. 
Here in the LMC is a system with similarities that suggest further investigation might be worthwhile.

\subsection{Magellanic Cloud inventory}

As a matter of interest, we list here the compact objects now known to be associated with Magellanic Cloud supernova remnants. The Large and Small Magellanic Clouds (LMC and SMC) contain $\sim 60$ remnants. About 45 of these are known to emit X-rays [Smith (web page, 2005), Williams et al. (2000), Inoue, Koyama, and Tanaka (1983), Filipovic et al. (1998)]. Thirty-four have so far been observed by Chandra and the results may be viewed in the online catalog at http://hea-www.cfa.harvard.edu/ChandraSNR/. Structure of the brighter remnants has been well-resolved with moderate observing times and much detail is visible. One can see shock waves, interior structure, clumpy material, central objects, and pulsar-wind nebulae. Chandra data have been used to compile Table 6, an inventory of X-ray emitting compact objects and PWN within Magellanic Cloud SNRs. Diffuse thermal radiation from the shells or interiors has not been included. Faint unresolved sources, for which no pulsations have been detected, could contain a contribution from a small PWN so the luminosities are listed under the header "PSR \& PWN". All luminosities have been corrected to the $0.5-10 \mathrm{keV}$ range. Ten of the 35 Magellanic Cloud SNRs observed by Chandra have PWN or compact objects. The luminosity range is a factor of $10^{4}$ and there is great variety of type; 1 SGR, 2 rotation-powered PSR/PWN, 1 CCO, 2 PWN with no associated compact object, 2 XRB (including this observation), and 2 unclassified faint objects. 
Table 6. Luminosity of Magellanic Cloud compact objects and PWNe

\begin{tabular}{|c|c|c|c|c|c|c|}
\hline Remnant & PSR or CCO & period & $\begin{array}{l}L_{X} \\
\text { PSR \& PWN }\end{array}$ & $\begin{array}{l}L_{X} \\
\text { compact }\end{array}$ & $\begin{array}{l}L_{X} \\
\text { pulsed }\end{array}$ & ref \\
\hline & & $(\mathrm{s})$ & $\left(\mathrm{erg} \mathrm{s}^{-1}\right)$ & $\left(\mathrm{erg} \mathrm{s}^{-1}\right)$ & $\left(\mathrm{erg} \mathrm{s}^{-1}\right)$ & \\
\hline SNR 0540-693 & B0540-69 & 0.050 & $2.1 \times 10^{37}$ & $1.7 \times 10^{37}$ & $0.4 \times 10^{37}$ & $(1)$ \\
\hline N157B & PSR J053747.3-691020 & 0.016 & $4.4 \times 10^{36}$ & $7 \times 10^{35}$ & $3 \times 10^{35}$ & $(2)$ \\
\hline N49 & SGR J052600.8-660436 & 8.04 & $1.0 \times 10^{36}$ & $1.0 \times 10^{36}$ & $<10^{35} ?$ & $(3)$ \\
\hline DEM L241 & CXOU J053600.0-673507 & - & - & $2 \times 10^{35}$ & - & this paper \\
\hline SNR 0453-685 & - & - & $6 \times 10^{34}$ & - & - & (4) \\
\hline DEM L316B & - & - & $\approx 5 \times 10^{34}$ & - & & $(5)$ \\
\hline $\mathrm{N} 23$ & J050552.3-680141 & - & - & $8 \times 10^{33}$ & - & $(6)$ \\
\hline SNR J0047.2-7308 & J004719.7-730823 & - & $\sim 3 \times 10^{33}$ & & - & $(7)$ \\
\hline N206 & - & - & $3 \times 10^{33}$ & $<1.5 \times 10^{33}$ & - & $(8)$ \\
\hline SNR J0127.7-7333 & SXP 1062 & 1062 & - & $7 \times 10^{35}$ & $1.4 \times 10^{35}$ & $(9)$ \\
\hline
\end{tabular}

(1) Kaaret et al 2001 (2) Wang et al 2001 (3) Kaplan 2002 (4) Gaensler et al 2003 (5) Williams \& Chu 2005

(6) Hughes et al 2006 (7) Seward et al 2012 (8) Williams et al 2005 (9) Hénault-Brunet 2011

\section{Conclusions}

This is an interesting supernova remnant, larger and more elongated than most. The absence of X-ray emission from the outer shock, the X-ray spectra, and the size all indicate an older remnant. Because of its age and location close to a strong H II region, radio emission is weak and undetectable. It contains a compact object with an O-star optical counterpart which is unusual for a supernova remnant. No non-thermal X-rays, as might originate in a PWN, are detected from the immediate vicinity of this source and the source luminosity and spectrum are consistent with that expected from a HMXB. The velocity of the O-star was observed to vary and a long period is suspected.

The diffuse X-ray spectrum from the remnant interior is enriched in $\mathrm{O}, \mathrm{Ne}$, and $\mathrm{Mg}$. This enrichment and the more-slowly evolving O-star companion imply that the supernova precursor star had a mass of $>25 M_{\odot}$. We note that the elongated envelope and the structure close to the compact source might have been formed by an SS 433-type pair of jets but there is no indication of these in the optical spectrum. 
Support for this work was provided by the National Aeronautics and Space Administration through Chandra Award Number GO1-12094 issued by the Chandra X-ray Observatory Center, which is operated by the Smithsonian Astrophysical Observatory for and on behalf of the National Aeronautics Space Administration under contract NAS8-03060. D.L.F. acknowledges support from NASA through the Harriett G. Jenkins Pre-doctoral Fellowship Program, and from the Vanderbilt-University of Cape Town Partnership. We thank Paul Green for an interesting discussion and information about background quasars. Sean Points supplied calibrated and aligned MCELS images. B. Furnish, J. Hood, C. McCarty, and T. Williams at Columbus State University helped with the X-ray spectral analysis.

\section{References}

Bamba, A., Ueno, M., Nakajima, H., Mori, K., \& Koyama, K. 2006, A\&A 450, 585

Blundell, K.M., Bowler, M.G. \& Schmidtobreick, L. 2008, ApJ 678L, 47

Chlebowski, T. 1989, ApJ 342, 1091

Crampton, D., Cowley, A.P.,Thompson, I.B. \& Hutchings, J.B. 1985, AJ 90, 43

Crawford et al. 1998, Mem.de Soc. Astron. Italy. 69, 95

Davis, R.D., Elliot, K.H. \& Meaburn, J. 1976, MmRAS 81, 89

Dickel, J. R., Mcintyre, V, Gruendl,,R., \& Milne, D. K. 2005, AJ 129, 790

Gaensler, B. M.; Hendrick, S. P., Reynolds, S. P., Borkowski, K. J. 2003, ApJ 594, L111

Hénault-Brunet, V. et al 2011, MNRAS 420, L13 
Henize, K. 1956, ApJS 2, 315

Hughes, A., Staveley-Smith, L., Kim, S., Wolleben, M., \& Filipović, M. 2007, MNRAS, 382,543 .

Hughes, J.P., et al. 2006, ApJ 645, L117

Jarius, D. 2002, Comparison of on-axis Chandra Observations of AR Lac to SAOSAC Simulations. CXO internal memorandum.

Kaaret, P, et al., 2001, ApJ 546, 1159

Kaplan, D.L., 2002, MmSAI 73, 496

Kim, Minsun, et al. 2007, ApJS 659, 29

Lockman, F.J., Blundell, K.M. \& Goss, W.M. 2007, MN 381, 881

Long, K.S., Helfand, D.J., \& Grabelsky, D.A. 1981, ApJ 248, 925

Margon, B., Grandi, S. A. \& Downes, R. A. 1980, ApJ 241, 306

Martins, F., Shaerer, D., \& Hillier, D.J. 2005, A\&A 436, 1049

Mathewson, D.S., Ford, V.L., Tuohy, I.R., Mills, B.Y., Turtle, A.J. \& Helfand, D. J. 1985, ApJS 58, 197

Mauch, T., Murphy, T., Buttery, H. J., Curran, J., Hunstead, R. W., Piestrzynski, B., Robertson, J. G., Sadler, E. M, 2003, MNRAS 342, 117

McGill Pulsar Group 2012, McGill SGR/AXP Online Catalog, http://www.physics.mcgill.ca/ pulsar/

Pollock, A., Corcoran, M., Stevens, I. \& Williams P. 2005, ApJ 629, 482

Rappaport S. A. \& Joss P. C. 1983, in: W. H. G. Lewin \& E. P. J. van den Heuvel (eds.) Accretion-driven X-ray Sources, (Cambridge: Cambridge University Press), 33 
Remillard, R. \& Mcclintock, J. 2006, ARA\&A 44, 49

Schmidtke, P. C., Cowley, A. P., Frattare, L. M., McGrath, T. K., Hutchings, J. B. \& Crampton, D. 1994, PASP 106, 843

Schmidtke, P. C.; Cowley, A. P.; Crane, J. D.; Taylor, V. A.; McGrath, T. K.;

Hutchings, J. B.; Crampton, D. 1999, AJ 117, 927

Seward et al 2012, Chandra Supernova Remnant Catalog, http://hea-www.cfa.harvard.edu/ChandraSI

Smith, R.C. and the MCELS team 1999, IAU Symposium 190, 28

Wang, Q. D., Gotthelf, E. V., Chu, Y.-H., Dickel, J. R. 2001, ApJ 559, 275

Woosley, S.E. \& Weaver, T.A. 1995, ApJS 101, 181

Williams, R.M. \& Chu, Y.-H. 2005 ApJ 635, 1077

Williams, R. M., Chu, Y.-.H., Dickel, J. R., Gruendl, R. A., Seward, F. D., Guerrero, M. A., \& Hobbs, G. 2005, ApJ., 628, 704 